\title{
ELECTROPHYSIOLOGICAL STUDIES ON THE WORKING MECHANISM OF MUSCLE WALLS OF THE STOMACH
}

\author{
Akio SATo* \\ Department of Physiology, School of Medicine, Niigata University, Niigata
}

The bio-electricity of muscle walls of the stomach has been investigated as one of the important fields of electrophysiological researches of visceral muscles. About fifty years ago STÜBEL ${ }^{20)}$ verified rhythmic action potentials in pigeon's stomach. In mammals the characteristic cycles of potential variation that accompanied rhythmic contractions of this organ were found by RichteR ${ }^{17}$ ), Alvarez and MAHONEY') and NiU ${ }^{14)}$. Recently BOZLER ${ }^{(i)}$ demonstrated the clearly-defined electric waves using specially devised electrodes and it was affirmed later by ICHIKAWA $^{1 \div 1}$.

In frogs or toads slow potential rhythm has been reported by TSCHERMAK ${ }^{24)}$, OGATA $^{151}$ and SASAKI ${ }^{1 \times)}$. On the other hand, GAUPP ${ }^{8)}$ confirmed that almost entire muscle walls except the cardiac and pyloric ends of frog stomach consisted exclusively of only circular fibers. It was further observed by the present author that this fact held good also for the stomach of toads especially in its middle part. If action potentials, therefore, are taken from these parts of the organ they are naturally kept from being mixed with those of longitudinal fibers which are common components in higher animals. For this reason, the study reported here was undertaken on toad to elucidate the working mechanism of the circular muscle coat of the stomach electrophysiologically.

\section{METHOD}

All experiments were performed on toad (Bufo vulgaris japonicus) in every season except summer. For the experiments in situ, the spinal cord was totally destroyed to avoid the electrical disturbance due to body moving. Then the animal was placed in dorsal position and the abdominal wall was resected enough to expose the stomach after depriving of the greater part of the abdominal skin. To lead off action potentials non-polarizable $\mathrm{Zn}-\mathrm{ZnSO}_{4}$ moist thread electrodes were attached to the stomach surface so that they would conform to its movements

Received for publication December 4, 1959.

* 值㷋明 ; 
without causing changes in contact (FIG. 5). A d.c. amplifier was used in conjunction with a cathode-ray oscilloscope with the Grass type kymograph camera.

On the circular-muscle strip (usually about $3 \times 40 \mathrm{~mm} . .^{2}$ ) which was removed from the middle zone of the stomach and got rid of the mucous membrane, simultaneous electrical and mechanical recordings were made using the Sugl's ${ }^{21}$ ) insulation box. The insulation box was provided with a separating wall having a hole at its lower center. After the muscle strip was fixed in the box, the smaller part in the minor Ringer pool was entirely pinched to serve as the indifferent pole to the other intact larger part in the major Ringer pool (FIG. 1). Then each end of the thread electrodes was immersed into the respective Ringer pool to lead off action potentials therefrom. In order to record the contraction wave oscilloscopically, the end of the intact part of the muscle strip was connected to an isotonic lever of which movements were converted into the resistance changes of water cylinder.

In case of nerve or muscle-strip stimulation, parallel narrow silver wire electrodes were used in connection with an electronic pulse stimulator. Room temperature was regulated between 18 and $22^{\circ} \mathrm{C}$. throughout the experiments.

\section{RESULTS}

(I) Experiments on circular-muscle strip

1) Observation by insulation method

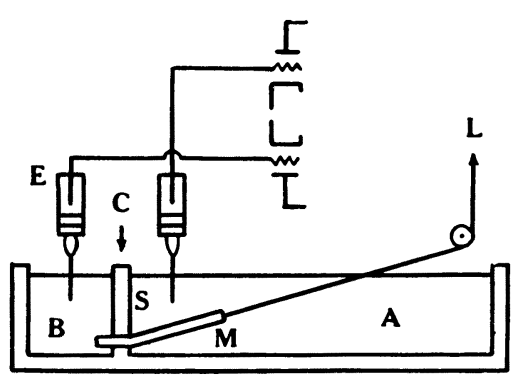

FIG. 1.

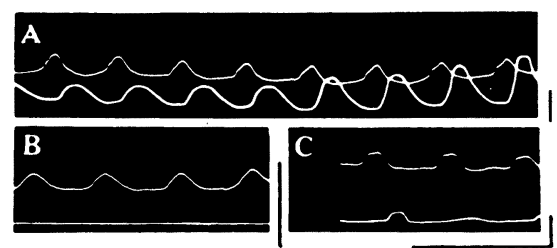

FIG. 2.

FIG. 1. (Left) Diagram illustrating the insulation method.

A : Major pool of Ringer. B: Minor pool of Ringer. C: Compression to secure the insulation between two pools. E: Nonpolarizable $\mathrm{Zn}-\mathrm{ZnSO}_{4}$ moist thread electrode. L: Lever. M: Muscle-strip. S: Separating wall.

FIG. 2. (Right) Various forms of simultaneous electrical and mechanical records from musclestrips obtained by the insulation method. Upper beam: Electrical record, lower beam: mechanical record. In electrical record upward deflection shows the negativity, in mechanical one the contraction. Note especially in $B$ that the electric activity is not necessarily accompanied by the mechanical one. Vertical bar $1 \mathrm{mV}$, horizontal bar: $1 \mathrm{~min}$. 
To clarify the fundamental problems concerning the relationship between muscle contraction and action potential studies were made on the muscle-strip using the insulation box (FIG. 1). Every automatic contraction was always accompanied by Iong lasting wave-like action potential of which magnitude ranged from 0.2 to $1 \mathrm{mV}$, duration 12 to $30 \mathrm{sec}$. and rate of rhythm 1.8 to 2.2 per min. (FIG. 2). The electrical manifestation usually preceded the mechanical wave and terminated as a rule before completion of the contraction (FIG. $2 A, C$ ). Although mechanical and electrical curves corresponded in shape, their magnitudes have not necessarily parallel relation to each other (FIG. $2 A, C$ ). When contraction waves were barely visible or totally absent, action potentials were often observed (FIG. $2 B$ ). Generally, the contraction height was apt to vary with time, while the potential height tended to remain rather unchanged.

Synchronism between mechanical and electrical events was disturbed by application of adrenalin into the major pool (FIG. $3 A, B$ ). Electrical records gave evidence of only irregular feeble pulsations at times accompanying various sizes of contractions. In addition to this, there developed a simultaneous downward shift of baseline lasting for 2 to $3 \mathrm{~min}$. that probably indicated the hyperpolarization of muscle cells.
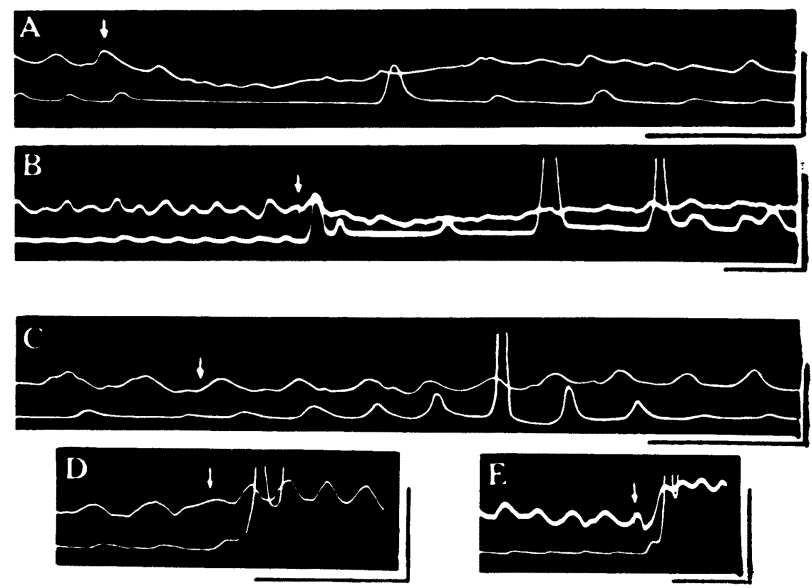

FIG. 3. Effects of drugs on potential waves of muscle-strips. At arrows drugs were introduced into the major pool containing $30 \mathrm{ml}$ of Ringer's solution. $A$ : Adrenalin $0.1 \mathrm{ml}, 3 \times 10^{-4} \%$. $B$ : Adrenalin 0.5 $\mathrm{ml}, 3 \times 10^{-4} \%$. $C$ : Acetylcholine $0.1 \mathrm{ml}, 3 \times 10^{-2} \%$. $D:$ Acetylcholine $0.5 \mathrm{ml}, 3 \times 10^{-2 \%}$. $E$ : Acetylcholine $1 \mathrm{ml}, 3 \times 10^{-2} \%$. Upper beam: Electrical record, lower beam : mechanical record. In electrical record upward deflection shows the negativity, in mechanical one the contraction. Vertical bar: $1 \mathrm{mV}$, horizontal bar: $1 \mathrm{~min}$.

Application of a small dose of acetylcholine produced the powerful automatic contractions which were left synchronous with the electrical events that proceeded 
without any observable modification (FIG. $3 C$ ). At higher concentrations, it was striking to find that these vigorous contractions were superimposed on the sustained tonic contraction, just corresponding to the fast electric undulation and its superposition on the maintained upward shift of baseline which suggested the depolarization of muscle cells (Fig. $3 D, E$ ).

2) On circular-muscle strip suspended in air

Bipolar recordings were made on the muscle-strip suspended in air by fixing its both ends with insulating material. FIG. $4 A$ shows that potential waves were traveling along the strip from one electrode to the other unidirectionally except the first wave which indicated that the conduction was blocked between them. This first monophasic curve bears a close resemblance to that obtained by the insulation method. As the author expected from the foregoing results, these electrical manifestations preceded the contraction waves observed microscopically.
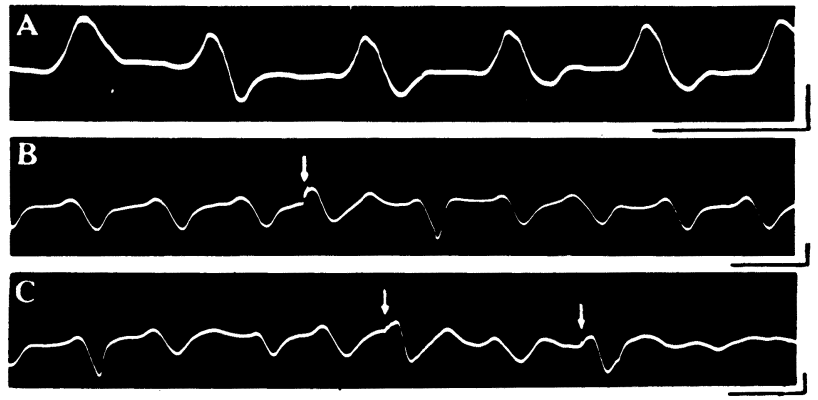

FIG. 4. Bipolar records from muscle-strips suspended in air to show the effects of direct electric stimulation. Inter-electrode distance: $7 \mathrm{~mm}$, distance between the stimulating and near-by recording electrodes: $6 \mathrm{~mm}$. $A$ : Control. $B, C$ : Effects of the stimulation. Arrow shows the pulse stimulation of about 1 sec. duration. Square pulse: Intensity $8 \mathrm{~V}$, frequency $10 \mathrm{c} / \mathrm{s}$, pulse duration $0.5 \mathrm{msec}$; on second stimulus in $C$ the intensity $24 \mathrm{~V}$. Vertical bar: $1 \mathrm{mV}$, horizontal bar: $1 \mathrm{~min}$.

Just suprathreshold stimulus was delivered to the end of the strip from which the potential wave originated. It produced a premature potential wave followed by the relatively rapid restoration of normal rhythmic waves (FIG. $4 \mathrm{~B}$, first stimulus in $C$ ). When the stimulus intensity was increased to three times the threshold value, the evoked wave was not followed by normal ones any more but only slight irregular variations (FIG. 4 second stimulus in $C$ ). The premature electric response seems to be essentially normal, but it is broadened in time and augmented in magnitude.

(II) Experiments in situ

1) Unipolar records from various parts of the stomach

Unipolar recordings were made when one electrode was attached to the stomach while the other to a piece of Ringer-soaked cotton on the thigh muscles. 
The experiment in FIG. 5 was performed on the same stomach which was motionless to the naked eye. The patterns of the monophasic potentials in situ coincide quite exactly with those of muscle-strip. In dogs Alvarez and MAHONEY ${ }^{2)}$ found out rhythmic gradient from cardia to pylorus. In this case, however, the rates of rhythm in various parts showed almost similar value of about 2 per min. as reported by OGATA ${ }^{15)}$ and SASAKI ${ }^{18}$. This agreement of the rate will be confirmed in the following section.

In many cases, amplitudes were roughly equal along the longitudinal axis of the organ except near the cardia and pylorus, but, in the transverse line potential gradients from the greater to the lesser curvature were clearly demonstrated (FIG. 6). This dominance of amplitude along the greater curvature was also observed in mammals by BOzLER ${ }^{6)}$.

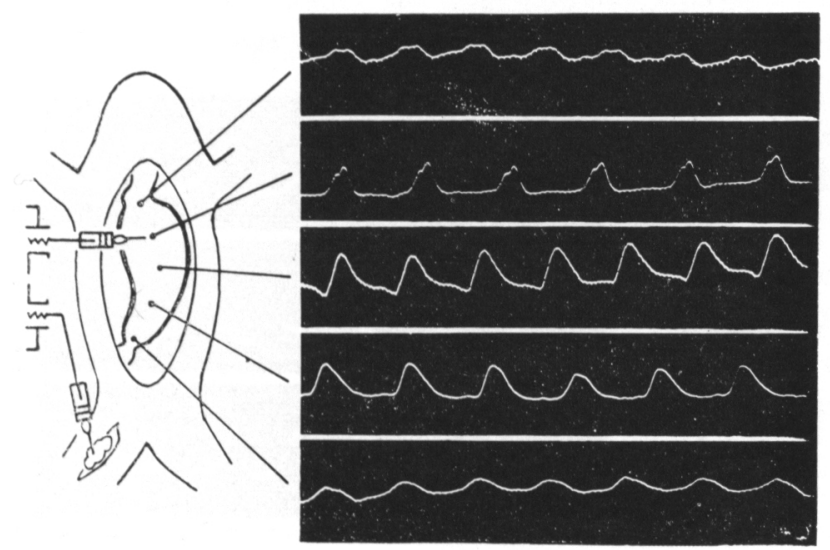

FIG. 5. Unipolar records from various parts of the same stomach in situ. Upward deflection shows the negativity. Tiny spikes in the lines especially in upper three show the contaminated electrocardiograms. Vertical bar: $1 \mathrm{mV}$, horizontal bar: $1 \mathrm{~min}$.

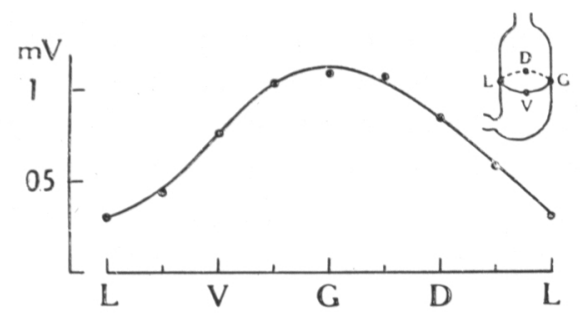

FIG. 6. Potential gradient along the line from the greater to the lesser curvature in a transverse direction in the middle stomach. Ordinate: Amplitude of the monophasic potential. Each point on curve represents the average amplitude of five successive waves. L: The lesser curvature, $\mathrm{G}$ : the greater curvature, $\mathrm{V}$ : ventral, D: dorsal as shown in the inset.

2) Bipolar records from the middle part of the stomach

As shown in FIG. 7 a series of recordings was carried out on the same stomach. At the start, unipolar recording was made with one electrode to point $\mathrm{X}$ and the indifferent to the thigh muscles in order to obtain the exact pattern of the potential 
changes that took place under the different electrode (FIG. $7 A$ ). In the second step, the indifferent electrode was immediately transferred to point $\mathrm{Y}$ located just transversely to point $\mathrm{X}$ keeping the first electrode undisturbed. With this arrangement clearly-defined diphasic potentials were obtained, which indicated the rhythmic travelings of potential waves along circular muscle band XY toward the lesser curvature (FIG. $7 B$ ). As the inter-electrode distance is known, it is possible to calculate the approximate velocity of the potential wave by measuring the time interval between the rising point and the peak of the initial phase. It was found to be $4 \mathrm{~mm}$. per sec. in average. In the third step, the transferred electrode was further moved to point $\mathrm{Z}$ located longitudinally in anal direction to point $\mathrm{X}$. As shown in FIG. $7 C$, the regular rhythm of undulating variation undoubtedly indicated the coincidence of the rates of rhythm between the potential waves along bands $X Y$ and $Z$. This relationship was always kept well no matter at what place on the stomach surface the point $\mathrm{Z}$ was chosen.
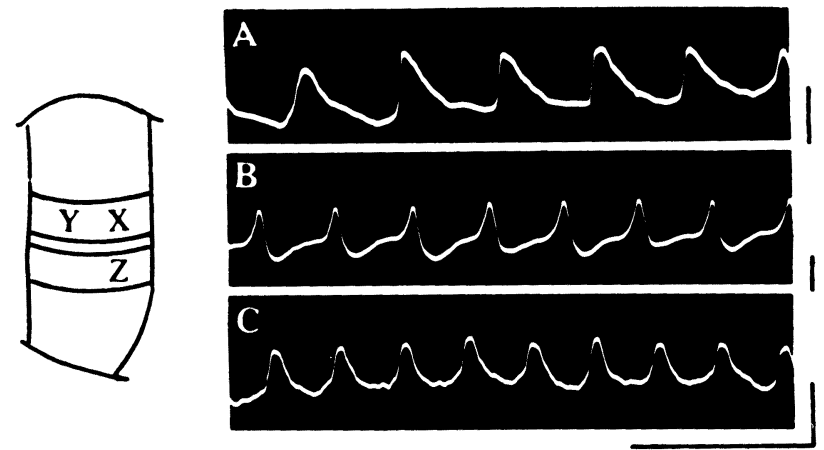

FIG. 7. A series of records from the same stomach.

$A$ : Recording electrode at point $\mathrm{X}$, indifferent electrode at the right thigh muscles. $B$ : Bipolar record from $\mathrm{X}$ and $\mathrm{Y}$. $C$ : Bipolar record from $X$ and $\mathrm{Z}$. Inter-electrode distance: $7 \mathrm{~mm}$. Vertical bar: $1 \mathrm{mV}$, horizontal bar: $1 \mathrm{~min}$.

In a few cases action potentials were obtained similar to those which were common in a dog's stomach in BozLER's research(i). The unipolar record showed a long continued state of negativity preceded by a characteristic initial quick phase (FIG. $8 \mathrm{~A}$ ). The bipolar potential consisted of three components, and it cannot be distinguished from corresponding record of cardiac muscle except for the fact that the duration of the whole complex is about 15 to $28 \mathrm{sec}$. (FIG. $8 A^{\prime}$ ). Incidentally it happened that the typical electrical manifestations occurred in pairs for many waves in succession as shown in Fig. $8 \mathrm{~B}$.

So far dealt with, the author has been struck by the extreme regularities of the rhythm and shape of the potential. But there is another fact regarding the spontaneous potentials that must be pointed out. Namely, their occasional sudden transformation from one wave to another took place. Records of this kind are 


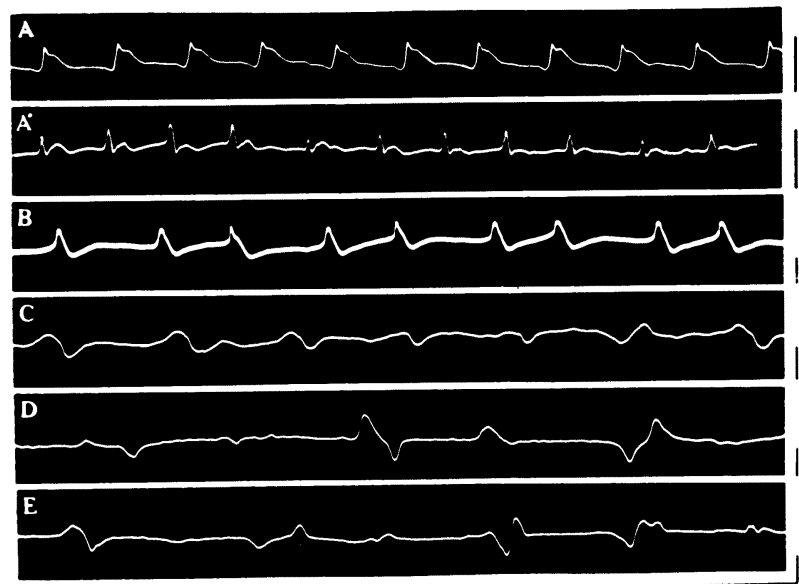

FIG. 8. Various types of wave processions taken with transverse arrangement of the electrodes. Inter-electrode distance: $7 \mathrm{~mm}$., except in $A$ which was the unipolar record of $A^{\prime}$. Tiny spikes contaminated in $A$ and $A^{\prime}$ show the electrocardiograms. Vertical bar: $1 \mathrm{mV}$, horizontal bar: $1 \mathrm{~min}$.

shown in FIG. $8 C$-E. FIG. $8 C$ indicates the development of reverse conduction. Waves in $D$ and $E$ were taken from the winter toad in hybernation. The low rates of rhythm and conduction would reflect the inactive condition of the organ. The waves appeared and vanished repeatedly. When appeared the direction of conduction was frequently reversed.

3) Action potential associated with muscle contraction

All experiments in situ above-mentioned were carried out on the stomachs that exhibited either very feeble or no movement at all to the naked eye. Therefore, to see how the potential waves correlate with peristaltic waves following observation was made. The electrodes were arranged transversely so that they were both reached by peristaltic waves at the same time. In FIG. $9 A$, large and small potential waves alternated at regular rhythm, and the large one was always associated with peristaltic wave of moderate strength coursing over the organ aborally at a velocity of about $0.5 \mathrm{~mm}$. per sec. It seems certain that these two kinds of waves are essentially the same, but the record indicates the fact that the wave is conducted more completely at the arrival of peristaltic wave. FIG. $9 B$ shows how finely a large potential wave in connection with a suddenly occurred peristaltic wave in the quiescent organ got into the rhythm of the recurring potential waves.

Sometimes a tonic contraction ring occurred and settled in the middle stomach for a few to several min. Potential waves from such rings were usually characterized by the considerable increase in their conduction velocity (FIG. $9 C, D$ ). However, the rate of rhythm remained unaffected together with the direction of conduction. When the ring disappeared, previous waves revived just as before (FIG. $9 C$ ). 
4) The liability to play role of pace-maker in the direction from the greater to the lesser curvature across the stomach

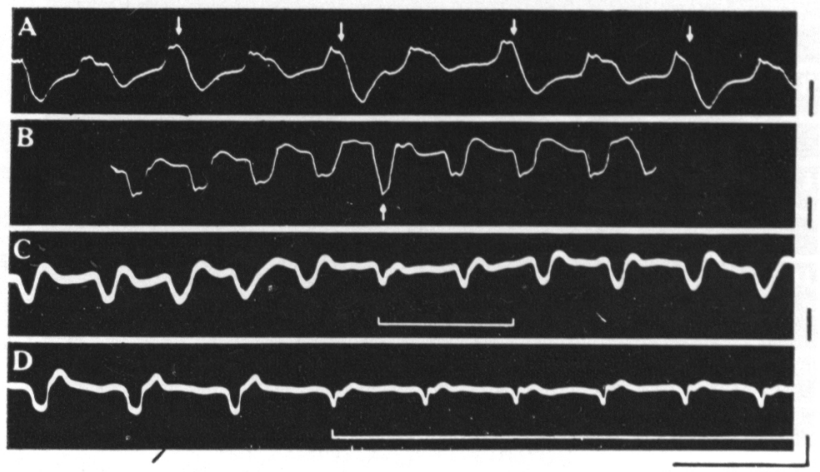

FIG. 9. Various wave shapes and their relationships to the muscle contractions observed macroscopically. The electrodes were arranged $7 \mathrm{~mm}$. apart transversely. $\boldsymbol{A}, \boldsymbol{B}$ : Potential waves indicated by arrows were associated with peristaltic waves. $C$ : A tonic contraction ring appeared and vanished in the middle of the record. $D$ : Appearance of a maintained tonic contraction ring. $\sqcup$ : Approximate duration of tonic contraction. Vertical bar: $1 \mathrm{mV}$, horizontal bar: $1 \mathrm{~min}$.

On the direction of conduction of the potential wave over the course of middle band the study was performed with successive attachments of the electrodes to its quarters (FIG. 10). The width of supposed band was taken as narrow as possible along circular muscles. In general, wave processions toward the lesser curvature were observed in more frequent cases than reverse ones both in ventral and dorsal halves of the ring. The ratio between them was about 3 to 1 estimated from 21 different cases. Further it was commonly noticed that the potential wave was conducted more perfectly in the greater curvature half than the other half of the ring where the conduction was frequently blocked. This tendency was especially exaggerated when the physiological bending of the longitudinal axis of the organ was augmented. FIG. $10 a-c$ represent three typical conduction patterns of waves most frequently observed, while Fig. $10 d$-f show the patterns less frequently seen. These findings would indicate the possibility that pace-makers, used here synonymously with the sites of origin of the potential waves not in anatomical but in functional sense, locate predominantly in the greater curvature. When the electrodes were arranged so that an estimated pacemaker be situated between them, small triphasic potentials with the same frequency were obtained, denoting that the impulses were discharged bilaterally at the same time (FIG. $10 a$ ). Sometimes reverse waves occurred in a certain quarter of the ring. FIG. $10 d$ indicates a new development of another pace-maker in addition to the previous one. In FIG. $10 e$ the possibility of shifting of a pace-maker is shown. Seeing from these shifting and haunting of the pace-makers, it is obvious that they 


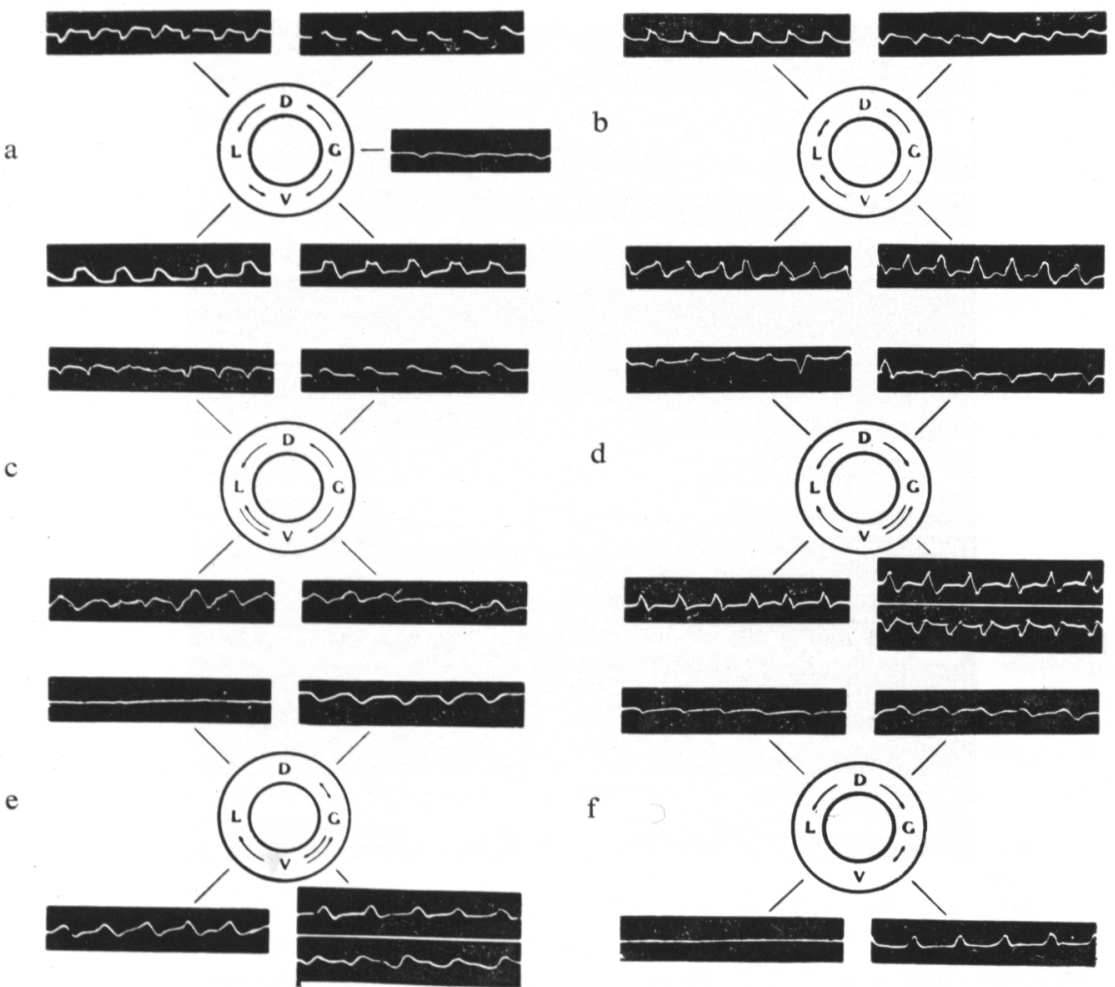

FIG. 10. Successive bipolar records from four quarters of middle band in situ. Arrows show the direction of wave conduction between the electrodes. Inter-electrode distance was two thirds the length of each quarter. L: The lesser curvature, $\mathrm{G}$ : the greater curvature, $\mathrm{V}$ : ventral pole, D: dorsal pole.

change easily in their sites and in their number as the time goes on. In short, there must exist the gradients of liability of the pace-maker to occur in the direction from the greater to the lesser curvature across the stomach.

5) Effects of nerve stimulation upon potentials

After attaching both electrodes transversely on the ventral middle wall, continuous electric stimuli were applied to vagosympathetic or splanchnic nerves.

a. Stimulation of vagosympathetic nerve: Stimuli were delivered to the peripheral cut end of the right vagosympathetic nerve at the cervical portion, which usually augmented the peristaltic movements in visual observation. Generally, they provoked a series of the potential waves with larger magnitude and higher rate of rhythm after a latency period of a few sec. (FIG. $11 A-D$ ). In many cases these waves were immediately replaced by the previous ones as soon as the stimulation was suspended (FIG. $11 C, D$ ). At the beginning of stimulation the enlarged waves depicted diphasic curves, but they were often soon transformed 


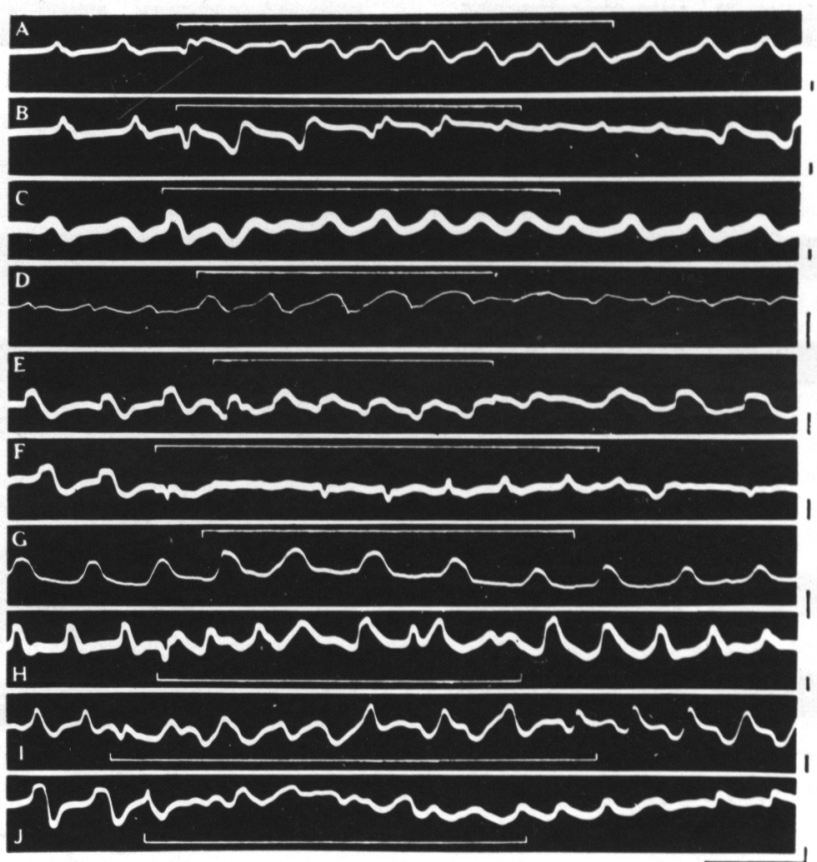

FIG. 11. Potential waves following stimulation of nerves. The electrodes were arranged across the ventral middle stomach. Interelectrode distance: $7 \mathrm{~mm}$., except in $G$ which was the unipolar record of F. $A-D$ : Stimulation of the peripheral cut end of the right vagosympathetic nerve. E-J: Stimulation of the splanchnic nerve. $\square$ : Duration of stimulation. Square pulse: Intensity $10 \mathrm{~V}$, frequency $10 \mathrm{c} / \mathrm{s}$, pulse duration $0.5 \mathrm{msec}$. Vertical bar: $1 \mathrm{mV}$, horizontal bar: $1 \mathrm{~min}$.

to the sinuous or odd shaped curves which might imply a gradual decrease in their conduction velocity (FIG. $11 C, D$ ). These effects perhaps suggest the faculty of this nerve to make synchronized the respective spontaneities of numerous muscle fiber units under electrode and participated the extra-fibers into this reinforced automatic activity.

b. Stimulation of splanchnic nerve: The nerve was stimulated at the running portion along celiacomesenteric artery. Two kinds of response patterns were obtained by whether the stimulation produces the prolonged tonic contraction or inhibits the movements of the middle stomach. The records of the former case are shown in Fig. $11 E-G$. In $E$, it is shown that a series of diphasic waves was at first disturbed but soon later followed by a procession of reverse waves with higher conduction velocity and rate of rhythm as long as the nerve was stimulated. In $F$, a small brief spike-like variation appeared following stimulation and after apparently silent phase of about $1.6 \mathrm{~min}$. this brief variation occurred again in succession and the polarity was inverted in the middle of the series. This brief 
spike-like potential should not be regarded as the mere miniature of the wave, but as the algebraic sum between two potentials which occur with mutual time lag of only about $3 \mathrm{sec}$. that is equivalent to the inter-electrodal conduction time of faster wave. The unipolar record of this case indicates that the shape as well as the magnitude of the monophasic potentials were not so influenced by the stimulation except the initial slight distortions (FIG. $11 \mathrm{G}$ ).

In the inhibitory cases, the irregularities of the amplitude and rhythm of the potential were worthy of note besides the disappearance of its diphasicity (FIG. $11 \mathrm{H}-J)$. These would denote that the wave of excitation was extremely suppressed in its conductivity and each functional unit, probably diminished in size, performed recurring excitations with its own rhythm suggesting the possibility of the units to take over the roles of pace-maker in themselves.

\section{DISCUSSION}

As mentioned above, a long lasting wave-like state of negativity was the most common shape of the monophasic potential of the toad stomach both in situ and in isolated strip. Recently OGATA ${ }^{15)}$ pointed out that the potentials from the same animal organ consisted of two components, slow phase and superimposed spikes on the former. In my experiments, however, such spike series were not found in any case except when the thread electrodes could not follow violent peristaltic rushes. In this respect analogous result was obtained for another toad visceral organ, the urinary bladder, by ICHIKAWA ${ }^{11)}$ who with similar thread electrodes demonstrated only slow electric variations contradicting the possibility of occurrence of spike series besides them which appeared in TAKAGI's report ${ }^{22}$. Reviewing through all the records the author noticed that as the conduction velocity of the potential wave increased the rising slope of the monophasic curve became steeper resulting in its gradual transformation from wave-like to right triangular shape with nearly the right angle corresponding to the steep rising slope. Though in a few cases, presumably, when the conduction was extra-fast there appeared the shape which was regarded as the further advanced stage of that transformation, i.e., initial quick phase with residual negativity commonly seen in Bozler's research. From these it is considered that the most primitive and fundamental pattern of the monophasic potential of this organ is a long continued wave-shaped curve.

As to the origin of potentials taken from alimentary tracts two theories, myogenic and neurogenic, have long been debated. But the former has received overwhelming supports from many workers. One of the facts supporting the neurogenic appears in BERKSON's report ${ }^{3-5)}$, namely, the fact that the dissociation of electric and mechanical activity occurred spontaneously and could be effected artificially by means of drugs, which led him to the hypothesis that the electric changes emanated from the intrinsic nervous plexus. In my experiment, also, this unparallelism of magnitudes between two manifestations was observed on 
muscle-strip preparation. But this fact itself cannot be the definite proof of the neurogenic theory. Other data reached are all on the side of the myogenic. Supposing the potential wave is neurogenic in origin, estimated magnitude of the potential to be produced in the intrinsic nervous plexus wholly shunted by surrounding tissues should be too small to be compared with the actual size. Besides this, extremely low conduction velocity of the wave along narrow muscle-strip should be properly ascribed to that of myogenic character and it would also support the opinion that smooth muscles work as syncytia.

Numerous works have been done on gastric movements by means of macroscopic observations or mechanical recordings. However, subvisual movements or subthreshold ones to mechanical recorders might be overlooked. Considering the fact that electric waves are still in full progress even when the gastric wall apparently fails to contract and that from differential recording approximate velocity as well as direction of their conduction can be determined, electrophysiological analyses should be taken as one of the excellent ways for the investigation of this organ. From the coincidence of rate of electric rhythm seen in entire stomach walls it is obvious that all the parts of the organ perform recurring excitations at the same frequency notwithstanding their motionless appearance to the naked eye. For the convenience of further discussion the author would like to call this rhythm basic potential rhythm. In the stomach of man TERAMOTO-23) reported the similar fact describing that rate of this basic rhythm was about 3 per min. These facts suggest the presence of a pace-maker that controls the automaticity of circular muscle bands of the walls, and it was located at the cardia according to OGATA ${ }^{15)}$ and SASAKI ${ }^{18)}$. On the other hand, peculiar gradients of potential and of liability of the pace-maker to occur in the direction from the greater to the lesser curvature would reflect the gradients of activity in the same direction across the stomach. On the basis of these findings, the general mechanism of peristaltic wave may be explained by assuming that the impulses emanated from the initial pace-maker at the cardia travel down the organ along the greater curvature predominantly, evoking the secondary pace-makers in each circular muscle band successively, which produce the corresponding basic potential waves in each band and certain proportion of them is accompanied by peristaltic contractions. The concept of the longitudinal conduction of the potential wave has been introduced by Prosser and SPERELAKIS ${ }^{16)}{ }^{19)}$ who observed that rings of circular muscle from cat intestine showed, in addition to circling conduction, waves which were transmitted along the long axis of the intestine.

The effects of nerve stimulation upon the movements of frog or toad's stomach have been worked out by AIKAWA ${ }^{1)}$, DIXON ${ }^{7)}$, ITAGAKI ${ }^{13)}$ and others. Quite recently $\mathrm{HATO}^{9) 10}$ ) clarified them by balloon-method, and described that the vagosympathetic stimulation brought forth only the augmentation in peristaltic character and the splanchnic mainly the augmentation in tonic character, but at times, combined with the inhibition of the movements. On the other hand studies 
on the effects of the nerve stimulation upon the electric waves were quite lacking. In another species these effects were observed in pigeon's stomach by STüBEL ${ }^{20)}$ and in dog's by RICHTER ${ }^{17)}$, but remarkable results were not obtained. In my analyses the vagosympathetic impulses increased the size and rate of rhythm of the potential while often decreasing its wave velocity. This seems to be analogous to the effect of peristaltic rush upon the basic potential waves and to the direct effect of stimulation of the muscle-strip upon its waves. The excitatory effect of the splanchnic nerve was characterized by increase in the conduction velocity, reversals of the direction of conduction, and increase in the rate of rhythm at times. These features differ considerably from those obtained in spontaneous tonic ring that exhibits only the augmentation of the conduction velocity. Commonly, however, for the development of tonic contraction it seems to be essential that the potential wave is conducted fairly more rapidly than usual. The frequent reversions of the wave direction rare to encounter in normal condition are worth suggesting the frequent hauntings as well as the shiftings of many newly evoked pace-makers along circular muscles, which, standing side by side with the peculiar inhibitory effect of this nerve, will play a certain important part on the nervous control of the muscular activity.

\section{SUMMARY}

Electrogastrogram was studied on the toad's stomach of which muscle walls consisted exclusively of circular fibers. The results obtained are as follows:

1) Every automatic contraction of the gastric muscle is always accompanied by the slow action potential both in situ and in isolated strip.

2) The greater part of the potential which usually precedes the muscle contraction terminates as a rule before the relaxation begins.

3) The rates of rhythm of the potentials are the same throughout the stomach. When peristaltic waves do exist, some of these basic potentials are associated with them.

4) For the development of tonic contraction it may be essential that the potential waves are conducted over the ring more rapidly than usual.

5) The gradients of potential and the liability of the pace-maker to occur in the direction from the greater to the lesser curvature are observed across the stomach. 6) Vagosympathetic stimulation will elicit a response pattern characterized by the increase in the magnitude and rate of rhythm of the potentials.

7) Splanchnic stimulation will elicit two kinds of response patterns, probably excitatory and inhibitory ones. The former is characterized by increase in the conduction velocity, reversals of the direction of conduction, and increase in the rate of rhythm at times; the latter by decrease in the conductivity and asynchronization of the potentials between respective functional units. 
The author wishes to express his sincere thanks to Prof. Koji UcH.zono for his kind advice and encouragement.

\section{REFERENCES}

1) Aikawa, T. On the innervation of the frog's stomach. Jap. J. med. Sci. III Biophysics 2 : 91-129, 1931-1933.

2) Alvarez, W. C. and Mahoney, L. J. Action currents in stomach and intestine. Am. J. Physiol. 58: 476-493, 1921-1922.

3) Berkson, J., Baldes, E. J. And Alvarez, W. C. Electromyographic studies of the gastrointestinal tract I. Am. J. Physiol. 102: 683-692, 1932.

4) Berkson, J. Electromyographic studies of the gastrointestinal tract III, IV. Am. J. Physiol. 104 : 62-72, 1933.

5) Berkson, J. Electromyographic studies of the gastrointestinal tract V, VI. Am. J. Physiol. 105 : 450-456, 1933.

6) Bozler, E. The action potentials of the stomach. Am. J. Physiol. 144:693-700, 1945.

7) Dixon, W. E. The innervation of the frog's stomach. J. Physiol. 28: 57-75, 1902.

8) Ecker, A. AND Wiedersheim, R. Anatomie des Frosches. 2 nd ed., revised by Gaupp, E., Abt. 3, Braunschweig: Friedrich Vieweg, 1904.

9) Hato, H. Effects of the electric stimulation of the peripheral cut end of rami communicantes and of the splanchnic nerves upon the movements of the frog's stomach. J. Physiol. Soc. Jap. $20: 388-396,1958$.

10) Нато, H. Effects of the electric stimulation of the peripheral cut end of the vagosympathetic nerves upon the movements of the frog's stomach. J. Physiol. Soc. Jap. 20: 401-406, 1958.

11) ICHIKAWA, S. On the action currents of the urinary bladder. Seitai no Kagaku 3: 275-278, 1951-1952 (in Japanese).

12) ICHIKAWA, S. AND BozLER, E. Monophasic and diphasic action potentials of the stomach. Am. J. Physiol. 182: 92-96, 1955.

13) ITAGakI, M. On the innervation of the stomach of the japanese frog. Jap. J. med. Sci. III Biophysics. 1 : 105-108, 1927-1930.

14) NIU, H. On the action currents of smooth muscles. Jiritsu-shinkei Zasshi. 5: 7, 133, 1956 (in Japanese).

15) OGATA, T. Electrophysiological study on the motility of the stomach. J. Kurume Medical Association. 17 : 324-339, 1954.

16) Prosser, C. L. AND Sperelakis, N. Transmission in ganglion-free circular muscle from the cat intestine. Am. J. Physiol. 187: 536-545, 1956.

17) Richter, C. P. Action current from the stomach. Am. J. Physiol. 67: 612-633, 19231924.

18) SASAKI, T. Studies on stomach peristalsis. J. Kurume Medical Association. 21: 860879, 1958.

19) Sperelakis, N. ANd Prosser, C. L. Mechanical and electrical activity in intestinal smooth muscle. Am. J. Physiol. 196 : 850-856, 1959.

20) STÜBEL, H. Der Erregungsvorgang in der Magenmuskulatur nach Versuchen am Froschund am Vogelmagen. Pflüg. Arch. ges. Physiol. 143: 381-394, 1912.

21) SugI, Y. Studies on the origin of the injury potential of muscle II. Jap. J. med. Sci. III Biophysics. $6: 331-368,1940$.

22) TAKagi, T. The electrogram of detrusor muscle in toad's bladder. J. Physiol. Soc. Jap. 19: 51-56, 1957. 
23) Teramoto, C. Studies on the human electrogastogram. Rinsyo to Kenkyü. 21: 438444, 1944 (in Japanese).

24) Tschermak, A. v. Bioelektrische Studien an der Magenmuskulatur. Pflüg. Arch. ges. Physiol. 175 : 165-186, 1919. 